\title{
Perceived Barriers to Asthma Therapy in Ethno-Cultural Communities: The Role of Culture, Beliefs and Social Support
}

\author{
Michele Shum ${ }^{1}$, Iraj Poureslami², Jing Liư ${ }^{3}$, J. Mark FitzGerald ${ }^{*}$ \\ ${ }^{1}$ University of John Hopkins, School of Public Health, Baltimore, MD, USA \\ ${ }^{2}$ University of British Columbia (UBC), Faculty of Medicine, Research Scientist at the Centre for Clinical Epidemiology and \\ Evaluation, UBC, British Columbia, Canada \\ ${ }^{3}$ British Columbia Cancer Agency, Smoking Cessation Project, Vancouver, Canada \\ ${ }^{4}$ Institute for Heart and Lung Health, The Lung Centre, 7th Floor Gordon and Leslie Diamond Health Care Centre, UBC, British \\ Columbia, Canada \\ Email: *mark.fitzgerald@vch.ca
}

How to cite this paper: Shum, M. Poureslami, I., Liu, J. and FitzGerald, J.M. (2017) Perceived Barriers to Asthma Therapy in Ethno-Cultural Communities: The Role of Culture, Beliefs and Social Support. Health, 9, 1029-1046.

https://doi.org/10.4236/health.2017.97075

Received: February 9, 2017

Accepted: July 9, 2017

Published: July 12, 2017

Copyright $\odot 2017$ by authors and Scientific Research Publishing Inc. This work is licensed under the Creative Commons Attribution International License (CC BY 4.0).

http://creativecommons.org/licenses/by/4.0/

(c) (i) Open Access

\begin{abstract}
Background: Adherence to therapy is integral to successfully managing asthma, which requires comprehension of what, when, and how to use medication and diligence in following management plan. Asthma patients from ethnic minority groups have more morbidity and reported filling their prescriptions less often. Limited information is available in Canadian literature on ethnic differences in their perceptions of asthma management. We aimed to document patient perceived adherence to asthma therapy among targeted ethno-cultural groups. Methods. We evaluated perceived barriers to therapy adherence, including: cultural beliefs and practices, patient/care-provider communication, self-management knowledge, and medication costs. We conducted a cross sectional study and interviewed 85 Chinese or Punjabispeaking adult asthma patients. Results. Lack of sufficient instructions from physicians, language/communication barriers, lack of skills on how to use inhalers, and high medication costs and medication side effects were most reported barriers to proper self-management practices. Most participants lived with others in the same household and reported high social support from home caregivers. The influence of family on self-management practices was obvious. Conclusion: Better understanding of patient needs, provision of culturally and linguistically appropriate education, and inclusion of home caregivers into the management practices are necessary to improve asthma outcomes in Chinese and Punjabi communities.
\end{abstract}

\section{Keywords}

Asthma, Medication, Family Involvement, Caregiver, Language, Culture, 
Educational Materials, Knowledge, Communication, Cost, Beliefs

\section{Introduction}

In Canada, asthma affects about 2.2 million adults and 0.8 million children (12 percent of Canadian population), and is highly prevalent in the mainstream population; in particular it tends to be less well controlled among individuals of lower socioeconomic status and low income families. Asthma is also prevalent in the Canadian recent immigrant communities and constitutes a disproportionate burden of disease compared to Canadian-born individuals. Asthma affects ethnic communities differently in terms of disease-related outcomes including morbidity, and mortality, when compared with the mainstream community [1]. Among these populations are Mandarin, Cantonese, and Punjabi speaking immigrants who are one of the largest and fastest growing cultural communities in Canada [2]. Within British Columbia, the Greater Vancouver Area (GVA) is the main location for these ethnic groups [3]. According to the 2010 Canadian Census data, these ethno-cultural communities make up 45\% of GVA's immigrant population [4].

Studies reveal that well controlled asthma requires adherence to therapy including self-management, proper use of medications, and following provider's instruction on treatment regimens [5] [6] [7] [8] [9]. However, less than 50\% of asthma patients adhere to medication recommended by their care providers [7] [10]. Poor adherence is especially prevalent in less educated and disadvantaged groups [11] [12] [13]. Non-adherence has many implications, including preventable disease progression, increased risk of exacerbations, reduced functional ability and quality of life, and an increased risk of death [14] [15] [16] [17] [18]. In contrast, adherence to therapy is associated with reduced mortality and a better quality of life [5] [6] [19] [20] [21] [22].

Previous qualitative research identified multiple factors that can affect ethnocultural patients' ability to learn and adhere to care plans and follow caregivers' prescribed therapy regimens, including patients' perception of their disease, type of treatment or medication, quality of patient-provider communication, costs of medications and treatment procedures, and obstacles to comprehension and uptake posed by language and cultural barriers [7] [9] [12] [23] [24] [25] [26]. Patients from ethno-cultural communities may face barriers to health care parity due to socio-cultural, behavioral, and psychological factors not addressed by the modern medical model. Consequently, these patient populations are disproportionately affected by health care disparities [13] [27].

Similar underlying determinants of proper self-management practices reported by different studies in mainstream and ethnic minority groups. While there are obvious gaps noted in both groups, including patient/care-provider communication, self-management knowledge, and medication costs, there is a knowledge gap in our understanding of how cultural beliefs and practices may 
effect therapy adherence among some ethno-cultural groups [28]. Through a cross-sectional study we investigated the link of these variables with self-reported adherence to asthma therapy and simultaneously examined the feasibility of involving family members, in the learning and self-management process. From our previous studies [29] [30] [31] [32], we observed that family members who normally give care to the patient (hereafter termed the "caregiver") are a key health-related social support for many patients in ethnic communities. The purpose of this paper is to summarize our findings in terms of identifying: 1) the issues and concerns re adherence to asthma therapy in Cantonese, Mandarin and Punjabi patients, 2) the role of culture, beliefs and family support in asthma self-management.

\section{Methods}

A participatory approach was applied to conduct the study and collect qualitative data from asthma patients in the target communities. This study was part of a larger intervention project aimed to develop and validate educational materials related to asthma management in Chinese and Punjabi communities [31]. The main objective of this study was to identify patients' perceived barriers to engage actively in the management of their asthma, including self-management practices. This article summarized the participants' self-reported attitudes, beliefs, and practices related to adherence to asthma therapy and challenges and barriers to practice disease management. The study was approved by the University of British Columbia Office of Research Ethics and was registered at ClinicalTrials.gov (trial number: NCT01474928).

\subsection{Participant Recruitment}

A purposive sampling method was applied to enrol asthma patients who had physician diagnosed asthma, who reported using asthma medications daily, were 21 years of age or older, had immigrated to Canada within the past 5 years, resided in GVA during the study period, and spoke Mandarin, Cantonese, or Punjabi. Exclusion criteria included patient under the age of 21 years, their diagnosis of asthma had not been made by a doctor in Canada, inability to attend the study session because of physical limitations, inability to provide informed consent, or could not communicate in Cantonese or Mandarin. We recruited 167 asthmatic adults of whom 35 participated in group sessions to help develop the study framework and a measurement tool, 40 participated in pilot testing this tool, and the remaining 87 subjects participated in the main study ( $85 \mathrm{com}$ pleted all study assessments). The 75 subjects who either participated in initial group sessions or pilot study did not participate in the main study. Study participants were identified and referred to the study by respiratory and family practice physicians in the collaborating clinics in the GVA. Bilingual facilitators contacted potential candidates by the phone and explained the study objectives, process, and risks in the patient's native language before recruiting them to study. They also outlined participants' rights to participate in the study and ob- 
tained signed consent in participants' native language.

\subsection{Measurement Tool Development}

Given the absence of a validated assessment tool in the target languages related to the objectives of this study, a qualitative questionnaire with 10 open-ended items (see Appendix A) to assess patients' comprehension and adherence to physician's instructions was developed in each target language. Initially, we invited 19 Mandarin and Cantonese and 16 Punjabi asthma patients to participate in four focus group sessions to assist in the development of the study framework and questionnaire. Patient's perceived concerns and challenges about asthma self-management were identified during the group sessions. It was very important to engage patients in the development of the study framework to reflect the needs and views of patients to generate meaningful and practical results. The information collected from patients enabled the team to develop useful assessment tool in English, which was then translated to Chinese Simplified and Traditional and Punjabi by professional translators. Back translation was applied to develop a measurement tool in each of the target community language. The assessment tool was pilot tested with 40 asthma patients from the target communities for clarity, relevancy, and comprehension of questions. In addition, for face and content validation, 10 community key-informants and health professionals from the target communities were invited to review and comment on the questions. Necessary modifications were applied based on patients' and key-informants' feedback.

\subsection{Data Collection}

Data was collected during in-person interviews and the assessment tool, was administered by trained facilitators, from the target communities. Before the data collection process began, each patient signed a consent form in their native language.

\subsection{Data Analyses}

To analyze the data, we applied four steps in order to identify specific themes: 1) systematically read the transcripts, reviewed the participants' responses, and compared and contrasted participant responses within groups to document emerging themes; 2) established categories and coding themes; 3 ) across group comparisons were made to ensure consistency in the coding; and 4) sorted responses into thematic categories. All responses were translated into English and then coded. Back translation was conducted to ensure accuracy of translation process. Initially two people conducted item coding and data extraction and discussed any disagreements. If agreed the changes were applied, otherwise a third member was involved to resolve any discrepancies and finalize the content. A professional qualitative analyser reviewed and commented on the qualitative data analysis process and necessary changes were applied accordingly. We classified recurring, emergent themes using continual comparison of the data. We added up the Mandarin and Cantonese patients in one group as "Chinese" to 
compare the objectives of interest with the "Punjabi" patients.

\section{Results}

\subsection{Participants' Characteristics and Medication Use}

42 Chinese and 43 Punjabi patients completed the study (aged 21 to 87 (Mean: 62.9 years, SD: 15.3 years), 42 males and 43 females). Thirty-nine participants (45.8\%) used only a reliever inhaler when needed, and 46 participants (54.2\%) used both reliever and controller inhalers. Among all participants, 59 (69.4\%) said they never received a written action plan and 22 (26\%) said they never received any oral or written instructions on how to use their inhaler from their doctor or from an asthma educator. More than $50 \%$ of patients who used both reliever and controller medications were unable to distinguish between the mode of action and purpose for the different inhalers (Table 1).

\subsection{Asthma Knowledge, Understanding, and Beliefs}

Participants' perspectives, knowledge, and beliefs about asthma medications, origin of their disease, and issues related to medication costs are summarized in Table 2. In general, the majority of the participants in both ethnic groups had difficulty understanding the reason for using reliever vs. controller medications and many of them blamed their doctor for their misinterpretation and indicated they never received instructions or an action plan in their language on how to correctly use inhalers and the reasons for using different medications. A suggestion made by some participants was a need to develop educational handouts and videos to be provided by an asthma educator or doctor from their community. They felt the focus of such discussions should include: medication differences, potential side effects of medications, correct use of inhalers. They also emphasized the need for educational materials that could be taken home.

Additional concerns included a belief that medication would be ineffective or cause an addiction. High cost of medication in Canada, especially compared to the cost of similar drugs in their native countries, was a concern mentioned by many Punjabi participants. Furthermore, there were some common misconceptions among older participants in terms of the origin of their asthma and causes of disease severity. While Punjabi participants believed using non-asthma drugs might be the main reason for having severe asthma, some Chinese participants indicated side effects of asthma medications maybe the reason for their disease getting worse. Finally, although many patients identified their doctor as a main source of receiving health information, language barriers in communicating properly with care providers was expressed by both Punjabi and Chinese participants. There was a feeling that their doctor was not sympathetic enough to their cultural beliefs and practices were a commonly expressed feeling especially by female participants.

\subsection{Patient Perceived Trust of Care Provider}

When asked whether they would adhere to a physician's instructions on how 
Table 1. Characteristics of the patients: patients who completed the interventions $(\mathrm{n}=$ $85)$.

\begin{tabular}{|c|c|}
\hline Characteristics & Value: $\mathrm{n}(\%)$ \\
\hline \multicolumn{2}{|l|}{ Sex } \\
\hline Male & $42(49.4 \%)$ \\
\hline Female & $43(50.6 \%)$ \\
\hline \multicolumn{2}{|l|}{ Education } \\
\hline Never attended formal school & $15(17.6 \%)$ \\
\hline Completed elementary School & $21(24.7 \%)$ \\
\hline Completed high school & $29(34.1 \%)$ \\
\hline Post high school education & $20(23.5 \%)$ \\
\hline \multicolumn{2}{|l|}{ Language } \\
\hline Chinese [Mandarin and Cantonese combined] & $42(49 \%)$ \\
\hline Punjabi & $43(51 \%)$ \\
\hline \multirow{2}{*}{ Age } & Mean: 62.9 years \\
\hline & SD: 15.3 years \\
\hline \multicolumn{2}{|l|}{ Family size } \\
\hline Live alone & $6(7.1 \%)$ \\
\hline Live with spouse & $9(10.6 \%)$ \\
\hline Live with young child/children & $11(12.9 \%)$ \\
\hline Live with adult child/children & $34(40 \%)$ \\
\hline Live with other adult family member(s) & $25(29.4 \%)$ \\
\hline \multicolumn{2}{|l|}{ Disease Severity } \\
\hline Stage I: Intermittent & $15(17.6 \%)$ \\
\hline Stage II: Mild persistent & $30(35.3 \%)$ \\
\hline Stage III: Moderate persistent & $26(30.6 \%)$ \\
\hline Stage IV: Severe persistent asthma & $14(16.5 \%)$ \\
\hline \multicolumn{2}{|l|}{ Current employment status } \\
\hline Employed & $18(21.2 \%)$ \\
\hline Unemployed & $25(29.4 \%)$ \\
\hline Retired & $37(43.5 \%)$ \\
\hline Volunteer job & $5(5.9 \%)$ \\
\hline \multicolumn{2}{|l|}{ Medications taken } \\
\hline Inhaled steroids & $23(27 \%)$ \\
\hline Long-acting bronchodilators & $7(8 \%)$ \\
\hline Combination medications & $46(54 \%)$ \\
\hline Short-acting bronchodilators & $48(56 \%)$ \\
\hline \multicolumn{2}{|l|}{ Asthma action plan } \\
\hline Never received a written action plan from doctor & $59(69.4 \%)$ \\
\hline Have received verbal information from doctor & $7(8.2 \%)$ \\
\hline Have received written action plan from doctor & $3(3.5 \%)$ \\
\hline No response (no data) & $16(18.8 \%)$ \\
\hline \multicolumn{2}{|l|}{ Can distinguish reliever and controller inhaler } \\
\hline Yes & $27(32 \%)$ \\
\hline
\end{tabular}


Table 2. Participants' perspectives and concerns about origin of diseases, asthma management, new medication development, and patient education.

\begin{tabular}{|c|c|}
\hline \multirow{3}{*}{$\begin{array}{l}\text { The origins of } \\
\text { disease }\end{array}$} & "What causes it? Is it genetic, and you can't change that?" \\
\hline & $\begin{array}{l}\text { "I like to know about the heritability of the first or second generation, if } \\
\text { hel she has a parent who is asthmatic, what is the outcome-the heritability } \\
\text { of a person having asthma too?" }\end{array}$ \\
\hline & $\begin{array}{l}\text { "What are the things that trigger asthma? How you develop it other than by } \\
\text { allergic. Is there a blood marker or something that makes you more at risk?" }\end{array}$ \\
\hline \multirow{5}{*}{$\begin{array}{l}\text { Patient education } \\
\text { program }\end{array}$} & $\begin{array}{l}\text { "I believe education plays an important part of disease management, and } \\
\text { education is communication". }\end{array}$ \\
\hline & $\begin{array}{l}\text { "I would like to learn more about my disease, how control triggers and how } \\
\text { to live more comfortably with it, possibly to expand my life". }\end{array}$ \\
\hline & $\begin{array}{l}\text { "I suggest education for the system [physicians] and patients-on both } \\
\text { sides". }\end{array}$ \\
\hline & $\begin{array}{l}\text { "Well, do even doctors - I mean a regular GP, how informed are they of } \\
\text { what's available? You get a GP who has got a patient diagnosed with asthma. } \\
\text { Do they know-does the doctor know enough to refer them on? It's pretty } \\
\text { few and far between to be honest with you". }\end{array}$ \\
\hline & $\begin{array}{l}\text { "The best way you get your health is to attend self-management education } \\
\text { sessions". }\end{array}$ \\
\hline \multirow{4}{*}{$\begin{array}{l}\text { Learning about } \\
\text { disease } \\
\text { management }\end{array}$} & $\begin{array}{l}\text { "I want to find the ways I can deal with my asthma personally without } \\
\text { having to involve the medical unless it's necessary sort of self- } \\
\text { management". }\end{array}$ \\
\hline & $\begin{array}{l}\text { "I want to find ways to be healthy and have the lifestyle that I want. I just } \\
\text { hope to find something". }\end{array}$ \\
\hline & $\begin{array}{l}\text { "What kind of strategies are there for self-management. diet control, what } \\
\text { type of exercise do I need to maintain that I m normal without having an } \\
\text { asthmatic attack, At what point should I ask my family member to take me } \\
\text { to the emergency". }\end{array}$ \\
\hline & $\begin{array}{l}\text { "I don't think my disease is completely under control. So I just want to be } \\
\text { able to control it. I want to be able to take deep breaths. I want to be able to } \\
\text { exercise and I don't want to have to think too hard about taking a breath. } \\
\text { It's very interesting to me to learn about these". }\end{array}$ \\
\hline \multirow{4}{*}{$\begin{array}{l}\text { Need to develop } \\
\text { helpful and cheap } \\
\text { medications }\end{array}$} & $\begin{array}{l}\text { "Government help to discover something to do with those lungs to make } \\
\text { them stronger whatever is left there". } \\
\text { "I want something that's going to be applicable to me as an asthmatic. } \\
\text { Something that is either going to help me to control my asthma or cure my } \\
\text { asthma if there is such a thing. Often you are just told what to do and you' re } \\
\text { told, take this drug, do this, do that. Maybe this drug is right for somebody } \\
\text { else but it s not the right one for me. P m told to take it but how do I know } \\
\text { that that there might be a better drug for me?" }\end{array}$ \\
\hline & $\begin{array}{l}\text { "I have not seen any new drug trials done in asthma at all. Surely new and } \\
\text { cheap medications can help to loosening the phlegm that you get. That's the } \\
\text { main thing that keeps you from breathing". }\end{array}$ \\
\hline & $\begin{array}{l}\text { "Maybe researchers can help develop better medications because current } \\
\text { medications are still not helping and are expensive". }\end{array}$ \\
\hline & $\begin{array}{l}\text { "Why companies not developing any new drugs that would help alleviate } \\
\text { the symptoms, while is affordable"? }\end{array}$ \\
\hline
\end{tabular}


they should use their asthma therapy, there were four major themes identified by the participants that would potentially improve adherence: 1) if they liked and trusted the physician (most likely with a doctor who communicated in their native language), 2) if the physician was honest with them in explaining the side effects of the medications, 3 ) if the physician took the time to listen to them and responded to their questions, and 4) if the physician understood the challenges the patient had with managing their asthma. The major issues and concerns regarding asthma information (accessing, communicating, understanding, using, etc.) are summarized below and also shown in Table 3.

\subsection{Perceived Barriers to Asthma Therapy}

Language and cultural barriers, lack of understanding care provider's instruction on self-management, and concern about side effects of medicine were identified by more than $50 \%$ of participants as barriers to taking asthma therapy. While we did not evaluate the impact of social support on adherence in this study, we noticed in many instances that caregivers' opinions influenced a patients' decision to fully adhere to therapy regiments: "My doctor told me I can take my inhaler 8 times a day but I take only 2 times because my son believes I can become somehow resistant to it'. In addition, the patients showed interest in learning more about the medications they were taking presented in their community language. They expressed their feeling that their cultural beliefs and practices should be somehow included in the educational information: "I am an elder and many people in my age group need relevant and trusted resources for their health concerns and questions; something is needed to be changed in Canadian health system in regard to culture and language issues".

\subsection{Link between Cultural Beliefs and Practices and Asthma Therapy}

There were some similarities and differences between the Chinese and Punjabi groups in their perceptions of the origins and implications of having asthma and self-management practices. For instance, a common belief amongst Chinese and Punjabi patients was that asthma is a communicable disease such as a common cold: " $A$ lot of people in my community believe that asthma is a communicable disease". Also many Punjabi participants expressed a feeling of being ashamed using an inhaler in public because they were concerned that others might believe they had transmissible infectious lung disease such as tuberculosis. In terms of smoking, there were different perceptions among the studied groups. While smoking was practiced openly and prevalent among Chinese patients, as they indicated smoking tends to be heavily promoted by the Chinese culture and helped to form a social connection with peers/friends and colleagues. In contrast, Punjabi patients mentioned smoking is not a practical habit in their community, specifically for women, and many indicated they prefer not to smoke in front of elders; expressing that it clashes with their religious and cultural norms. These beliefs and lack of understanding may indirectly impact patients' decision 
Table 3. Patients' concerns and comments about their challenges related to their asthma management.

\begin{tabular}{|c|c|}
\hline Factor & Patients statements \\
\hline Access issues & $\begin{array}{l}\text { "If we can have access to sources in our own language, we will be better able to get more and relevant } \\
\text { information, especially those of us who don't know English very well". } \\
\text { "It is difficult to access information here, because it is in other languages and if one knows a little bit of English } \\
\text { could (sic) just understand some words. I, myself, have to check the dictionary or ask my relatives. I think that } \\
\text { is a barrier, the language, I mean. It is complicate (sic)". } \\
\text { "The information I try to access here is very difficult to understand because they were written in English. I } \\
\text { normally ask my daughter to translate the information for me". } \\
\text { "I can say majority (sic) of the printed information is in English and the medical terminologies are very } \\
\text { difficult for me to understand". }\end{array}$ \\
\hline $\begin{array}{l}\text { Communication } \\
\text { Issues }\end{array}$ & $\begin{array}{l}\text { "I have never received any verbal or written explanation from my doctor and I have never heard about asthma } \\
\text { action plan". } \\
\text { "I do not know how to properly use my inhaler. My doctor never showed me how to use it". } \\
\text { "We see a little of negligence from physicians. When one makes a comment about a disease, they think that we } \\
\text { are educating them" } \\
\text { "My family doctor should make (sic) a good communication with me as a patient from different culture and } \\
\text { respects my beliefs, feelings, and traditions". } \\
\text { "I always concern that whether my family doctor is given enough time to me talking about my problem". } \\
\text { "The information provided to me by my doctor was not useful ... I felt I couldn't ask more questions because } \\
\text { he was very closed for (sic) further communication". } \\
\text { "The information given by my doctors is verbal instructions, and sometimes is difficult for me to understand". } \\
\text { "I think understanding is more important than speaking with the same language. You may visit a doctor who } \\
\text { speaks your home language, but s/he does not completely understand you or communicate properly with you } \\
\text { and vice versa". }\end{array}$ \\
\hline
\end{tabular}

"My primary source of health information is my family doctor. I am satisfied with my doctor because we speak the same language and I trust him".

"The doctor I visited different (sic) times for my condition wasn't helpful at all for me. I wanted to ask him about the asthma and its symptoms but he was very busy. It wasn't easy for me to speak with him".

Language and culture issues

"Some of the information I receive from my doctor are not culturally appropriate".

"...when the specialists explain about healthy or balance (sic) diet, the word health or balance (sic) diet have different meanings for me and are not specifically suitable for Chinese people".

"I want to say that (sic) language barrier is one thing, communication with doctor is something else. I think the information I got from my doctor didn't fulfill my needs".

"In Canada, I cannot find much information about my asthma condition because of language barrier (sic)".

"What are the side-effects from the medications?"

"The medications have to be prescribed here and are not selling (sic) over the counter. I remember when we first arrived in Canada we did not have the (sic) medical access card and it was the first barrier to services. I had

General concerns to bring the medicine in my luggage".

"I am always worried that I would be able to do nothing when I have asthma attack".

"I would like to see that there is a health care system [in Canada] where newcomers who cannot speak English well still receive needed services and help". 
to involve in self-care of their chronic illness.

\section{Discussion}

This study examined the issues and concerns of asthma management in Cantonese, Mandarin and Punjabi patients. Our findings suggest that lack of communication between patient and care provider, concerns about cost of medication, and beliefs about side effect of medications were perceived as major barriers to adherence by study participants. Such challenges and skill barriers in the target communities may increase their risk of poor outcomes. These findings confirm the results of studies that assessed the effectiveness of educational intervention on asthma outcomes in minority communities, in particular focusing on improvement of proper communication between patient and care provider and modifying disease and medication-related misunderstandings [6] [9] [33]. Indeed in our own study using appropriate educational material we saw improved asthma outcomes [30] [31].

Although we focused on overlapping themes that emerged within the studied ethno-cultural groups, it is important to identify other factors that may influence participants' beliefs and perceptions about disease management. One of the challenges identified in this study is related to "access" to relevant information to the target communities. A suggested approach to improving accessibility of information and services is developing educational interventions and directly involving patients and caregivers [34].

Another challenge was communication barriers between patients and providers. Many participants spoke of their perceptions in regards to how doctors do not respect their culture, do not respond to their needs because of being too busy, and do not speak their language. The participants indicated that due to the limited number of care providers from their communities who can communicate in their mother tongue they were facing communication isolation in the clinical encounters with English-speaking doctors. Similar issues have been reported in other studies [23] [35] [36]. Lack of proper communication resulted in patients' feeling insufficiently instructed as many participants reported having received instructions once or not at all, and complained they never had an opportunity to receive informative education on how to use their inhalers from their care providers. These communication issues significantly complicate selfmanagement practices [1]. Future medical encounters should include avoiding medical terms and using plain language by the care provider, and ideally having a fluent speaker adult family member, as navigator, when communicating with patients who have limited English proficiency [37] [38] [39] [40]. Providers should also seek to understand patients' cultural beliefs and practices towards the illness. Such practices will allow a more realistic representation and rigorous insights into making the doctor-patient communication process more culturally competent [27] [41].

The last but not least challenge identified by participants considered to be a barrier to therapy adherence was the cost of medications prescribed by provid- 
ers. Different studies have shown involvement in the care process and trust between patient and care provider are strongly correlated to a willingness to pay for medications [9] [24]. As the Canadian health care system does not provide universal insurance coverage for medications, cost is a significant factor for most patients in both mainstream and ethnic minority communities and it reduces people's capacity to achieve consistent adherence [18] [20] [21]. The total costs for asthma treatment in Canada are likely much higher than many other developed countries [5] [21]. A recent study has shown that around $64 \%$ of asthma patients in British Columbia (BC) had poorly controlled asthma and this group was responsible for $90 \%$ of the overall cost of asthma care in BC [42]. This financial burden was significant among participants of our study as more than $55 \%$ of study subject were over 65 years old and over $60 \%$ were unemployed or retired and relying on social insurance. Most of these participants indicated that high cost of asthma medication was a barrier to regular use of medication and suggested the possibility of reimbursement of costs by the government for low-income families [43]. In our study, we found that Punjabi patients were more concerned about the cost of the medications, while Chinese patients focused on the side effects of prescribed medications. Under dosing of medication was common, among both groups, but for different reasons. Punjabi participants indicated they often bought cheaper generic asthma medications from their homeland. Such practice may be related to patient's level of education and socio-economic status, as well as disease-related beliefs and cultural norms, as is shown in other studies [44]. Participants burdened by medication costs were further reluctant to bear the cost of asthma medications based on their limited knowledge of their condition and the benefits of proper medication use.

Although the literature of the family's role in supporting individuals with chronic disease, including asthma, is inconclusive about the effectiveness of family-oriented disease self-management interventions, we identified asthma management in Chinese Mandarin and Cantonese communities is a collective decision made by the patient and family members. For instance, a major influence of family member on self-management and medication adherence was facilitating the accessing and using asthma services as well as use or decline using medication, as prescribed. At various times throughout the study, we observed that many family members provided caretaking roles yet none were included in treatment plans or self-management practices. Despite this, the integration of caregivers in disease management was recognized by most participants in this study as often been lacking. Many revealed an inability to remember physicians' instructions without their caregiver assistant and felt a distinct advantage when experiencing a worsening of their asthma when the care provider could provide the information. We believe to ensure proper adherence to therapy, including social support in self-management such as family members with a better command of English, can play a key role in improving access to needed health information. Such a consideration will enhance our understanding of culturally-based perceptions as well as useful approaches needed to formulate and de- 
sign study objectives and goals [45] [46] [47] [48] [49].

\section{Limitations}

There are some limitations in our study. First, although we were interested in assessing family support on health outcomes we did not systematically measure family impact on the outcomes of interest, as we noticed the importance and the influence of family support on self-management practices during course of the study. Therefore, we suggest involving family members who normally provide care to the patient (named immediate care giver at home) should participate in the clinical assessment and share their thoughts and perception about asthma self-management. Next, we believe that our sample size does not have the breadth to generalize certain ethno-cultural communities' cultural norms and health behavior, as the study patients may not be the representative of all asthma patients in the target communities. Furthermore, reliance on self-reported adherence may leave the results susceptible to response bias, such as social desirability bias. However, completion of the study by $97 \%$ of the enrolled subjects confirms that adults with asthma from Punjabi, Cantonese, and Mandarin communities are interested in taking an active role in their self-management and in participating in a health education intervention that can be integrated into routine asthma care. Finally, although our aim was to show the link between family support and medication adherence in asthma patients from Mandarin and Cantonese communities in Greater Vancouver area, we believe that our patients, who were recruited from collaborative clinics, do not have the breadth to generalize Chinese ethno-cultural communities' cultural norms and health behavior in British Columbia of Canada due to the nature of the study. It is not clear to what extent the patients studied are representative of all patients in the target communities using asthma medication. Therefore, further research a larger sample size is needed to apply practical approach to recruit patients from larger Mandarin and Cantonese speaking communities in Canada to generate more inclusive and generalizable results. However, we believe the results of our study may well hold for those patients who were inclined to participate in such an educational intervention.

\section{Conclusion}

Social context, cultural beliefs and practices, and family support play distinct and significant roles in asthma control among Chinese and Punjabi patients, many of whom are less likely to benefit from medical directives due to language and cultural barriers. In a health system that typically favors a genomics aspect of medicine, patients are vulnerable to feeling misunderstood and diminished by healthcare providers [50]. Different patient groups may experience different barriers to adherence, but fear of side effects, lack of proper communication with care provider, and costs of medications were important to many participants in our study. The vast majority of the study participants expressed that language and cultural barriers as well as doctor-patient communication are the major 
problems when trying to connect with Canadian health system. Many participants indicated they prefer to visit a care provider from their own community who can speak their native language. Although medical interpreters are crucial in improving access to needed health care services of patients who do not speak the mainstream language, but engaging family caregivers, specifically younger generation who is fluent in the mainstream language, is crucial to the success of disease management.

Our findings show the usefulness of involving patient and caregiver in the learning process and engaging in self-management practices while providing culturally and linguistically appropriate information. Such practice can empower patient to develop and strengthen a sense of self-efficacy and confidence in managing their asthma. Patient education is the major intervention to promote self-management practices. However, to be effective, patients' beliefs, perceptions, and cultural norms should be addressed in educational interventions for ethnic minority communities. Further, health education promoters should incorporate caregivers' and patients' perspectives about asthma therapy into patient education programs [8]. Such integration can promote self-management practices among patients. We anticipate the gained knowledge from this study can be applied in the future research to promote asthma self- management, and engage patients in the development and implementation of relevant interventions.

Practical implication: Our study has shown that to improve asthma selfmanagement and therapy adherence, a provision of educational intervention to improve disease-related knowledge should be complemented with behavioral modification and empowerment techniques to enhance patients' self-efficacy skills. To be successful, asthma self-management in ethnic communities, in particular Mandarin and Cantonese groups, does require a multifaceted approach that incorporates patients' and family caregivers' opinion and perspectives in designing and implementing strategies to empower patients to become informed individuals. It is also essential to improve patients' confidence in their ability to follow physician instruction about medication adherence, as a major predictor of successful disease management practices. A fully informed patient is capable to plan and achieve self-care practice goals. The results of our study indicate that side effect, costs, cultural beliefs and practices, and social influence (such as family-oriented modeling) can be important factor in medication adherence. Therefore, appropriately developed self-management interventions, with direct involvement of patient and family members, can lead to increased patient's disease management competency overtime. Such interventions should emphasis on three main factors to promote medication adherence in Mandarin and Cantonese communities: improve patient understanding of physician instruction by improving patient-physician proper communication; correct patient's misconceptions about medication side effects and misperceptions about medication efficacy; and involve family members (as immediate caregivers at home) in disease management practices. 


\section{Acknowledgements}

The authors would like to thank the community members, facilitators, immigrant serving agencies, and key-informants from Punjabi and Chinese communities in the Greater Vancouver Area who helped us from the initial steps of this research. We would also like to kindly appreciate the Chinese and Punjabi physicians and health professionals for their contribution in the study design, material development, and patient recruitment. The links to audio-visual materials developed in the study are:

Punjabi community video

http://www.youtube.com/watch?v=qto8Sx2VIBM

Punjabi knowledge video

http://www.youtube.com/watch?v=SuS-YDT9vcE

Chinese community video 1

http://www.youtube.com/watch?v=LmT1qunrs1U

Chinese community video 2

http://www.youtube.com/watch?v=UhPkF6RDInU

Chinese knowledge video

http://www.youtube.com/watch?v=BJCOQK9EZ1k

\section{Funding Information}

This project was funded by the Canadian Institutes of Health Research (CIHR) and partly from the Institute for Heart and Lung health at The University of British Columbia, Vancouver.

\section{Conflict of Interest}

The authors have disclosed no conflicts of interest.

\section{References}

[1] Partridge, M.R. and Hill, S.R. (2000) Enhancing Care for People with Asthma: The Role of Communication, Education, Training and Self-Management. 1998. World Asthma Meeting Education and Delivery of Care Working Group. The European Respiratory Journal, 16, 333-348. https://doi.org/10.1183/09031936.00.16233400

[2] Statistics Canada. Ethnic Diversity and Immigration. 2011 National Household Survey. 2013. Available at:

https://www12.statcan.gc.ca/nhs-enm/2011/as-sa/99-010-x/99-010-x2011001-eng.cf $\underline{\mathrm{m}}$

[3] Statistics Canada. Immigrants' Perspectives on Their First Four Years in Canada: Highlights from Three Waves of the Longitudinal Survey of Immigrants to Canada. 2008. Available at:

http://www5.statcan.gc.ca/olc-cel/olc.action?objId=11-008-X20070009627\&objType $=47$ \&lang=en \&limit $=0$

[4] B.C. Immigration Trends 2009 Highlights. British Columbia Provincial Nominee Program 2016. Available at:

https://www.workbc.ca/getmedia/a98dc2e6-d41a-4281-ae2d-0151f2111485/immigra tiontrends2009.pdf.aspx 
[5] Bender, B.G. and Rand, C. (2004) Medication Non-Adherence and Asthma Treatment Cost. Current Opinion in Allergy \& Clinical Immunology, 4, 191-195. https://doi.org/10.1097/00130832-200406000-00009

[6] Ulrik, C.P., Backer, V., Søes-Petersen, U., Lange, P., et al. (2006) The Patient's Perspective: Adherence or Non-Adherence to Asthma Controller Therapy? Journal of Asthma, 43, 701-704. https://doi.org/10.1080/02770900600925569

[7] Horne, R. (2006) Compliance, Adherence, and Concordance: Implications for Asthma Treatment. CHEST, 130, 65S-72S.

[8] Clark, N., Lachance, L., Milanovich, M.F., Stoll, S. and Awad, D.F. (2009) Characteristics of Successful Asthma Programs. Public Health Reports, 124, 797-805. https://doi.org/10.1177/003335490912400606

[9] Piette, J.D., Beard, A., Rosland, A.M. and McHorney, C.A. (2011) Beliefs That Influence Cost-Related Medication Non-Adherence among the "Haves" and "Have Nots” with Chronic Diseases. Patient Preference and Adherence, 5, 389-396. https://doi.org/10.2147/PPA.S23111

[10] Tettersell, M.J. (1993) Asthma Patients' Knowledge in Relation to Compliance with Drug Therapy. Journal of Advanced Nursing, 18, 103-113. https://doi.org/10.1046/j.1365-2648.1993.18010103.x

[11] Gallefosse, F. and Per, S.B. (1999) How Does Patient Education and Self-Management among Asthmatics and Patients with Chronic Obstructive Pulmonary Disease Affect Medication? American Journal of Respiratory and Critical Care Medicine, 160, 2000-2005. https://doi.org/10.1164/ajrccm.160.6.9901028

[12] Halm, E.A., Mora, P. and Leventhal, H. (2006) No Symptoms, No Asthma: The Acute Episodic Disease Belief Is Associated with Poor Self-Management among Inner-City Adults with Persistent Asthma. Chest, 129, 570-580. https://doi.org/10.1378/chest.129.3.573

[13] Cabana, M.D., Lara, M. and Shannon, J. (2007) Racial and Ethnic Disparities in the Quality of Asthma Care. Chest, 132, 810S-817S. https://doi.org/10.1378/chest.07-1910

[14] Lahdensuo, A. and Muktari, A. (1986) Bronchodilator Effects of a Fenoterolmetered Dose Inhaler and Fenoterol Powder in Asthmatics with Poor Inhaler Technique. European Journal of Respiratory Diseases, 68, 332-335.

[15] Lindgren, S., Bake, B. and Larsson, S. (1987) Clinical Consequences of Inadequate Inhalation Technique in Asthma Therapy. European Journal of Respiratory Diseases, 70, 93-98.

[16] Vidgren, M., Kirkkainen, A., Karjalainen, P., et al. (1988) Effect of Dry Powder Inhaler Design on Drug Deposition in the Respiratory Tract. International Journal of Pharmacotherapy, 42, 211-216.

[17] Karsenty, H. and Thiriet, C. (1993) Correlation between Misuse of Metered DoseInhalers and Severity of Asthma. The European Respiratory Journal, No. 6, 147.

[18] King, D., Earnshaw, S.M. and Delany, J.C. (1991) Pressurised Aerosol Inhalers: The Cost of Misuse. The British Journal of Clinical Practice, 45, 48-94.

[19] Public Health Agency of Canada (2002) Economic Burden of Illness in Canada: Chronic Respiratory Diseases. Ottawa. http://www.phac-aspc.gc.ca/cd-mc/crd-mrc/index-eng.php

[20] Bahadori, K., Doyle-Waters, M., Marra, C., Lynd, L., et al. (2009) Economic Burden of Asthma: A Systematic Review. BMC Pulmonary Medicine, 9, 24.

[21] Sadatsafavi, M., Lynd, L.D., Marra, C.A., et al. (2010) Direct Health Care Costs Associated with Asthma in British Columbia. Canadian Respiratory Journal, 17, 74-80. 
https://doi.org/10.1155/2010/361071

[22] Global Initiative for Asthma (GINA) (2017) 2017 GINA Report, Global Strategy for Asthma Management and Prevention.

http://ginasthma.org/2017-gina-report-global-strategy-for-asthma-management-an d-prevention/

[23] Beck, R.S., Daughtridge, R. and Sloane, P.D. (2002) Physician-Patient Communication in the Primary Care Office: A Systematic Review. JABFP, 1, 25-38.

[24] Chen, Y., Tang, M., Krewski, D. and Dales, R. (2002) Association between Income Adequacy and Asthma Prevalence in Canadians. Proceedings of Statistic Canada Symposium Modeling Survey Data for Social and Economic Research.

[25] Raynoremail, D.K., Savage, I., Knapp, P. and Henley, J. (2004) We Are the Experts: People with Asthma Talk about Their Medicine Information needs. Patient Education and Counseling, 53, 167-174.

[26] Sawyer, S.M. and Shah, S. (2004) Improving Asthma Outcomes in Harder-to-Reach Populations: Challenges for Clinical and Community Interventions. Paediatric Respiratory Review, 5, 207-213.

[27] Zambrana, R.E., Molnar, C., Munoz, H.B. and Lopez, D.S. (2004) Cultural Competency as It Intersects with Racial/Ethnic, Linguistic, and Class Disparities in Managed Healthcare Organizations. The American Journal of Managed Care, 10, SP37SP44.

[28] Shaw, J.S., Huebner, C., Armin, J., et al. (2008) The Role of Culture in Health Literacy and Chronic Disease Screening and Management. Journal of Immigrant and Minority Health, 11, 460-467. https://doi.org/10.1007/s10903-008-9135-5

[29] Poureslami, I., Nimmon, L., Doyle-Waters, M. and FitzGerald, J.M. (2012) Using Community-Based Participatory Research (CBPR) with Ethno-Cultural Groups as a Tool to Develop Culturally and Linguistically Appropriate Asthma Educational Materials. Journal of Diversity in Health and Care, 8, 203-215.

[30] Poureslami, I., Nimmon, L., Doyle-Waters, M., Shum, J. and FitzGerald, J.M. (2013) Hearing Community Voice: Methodological Issues in Developing Asthma SelfManagement Educational Materials for Immigrant Communities. In: Ethnicity. Theories, International Perspectives and Challenges Charles Agyemang, Collins O. Airhihenbuwa and Ama De-Graft Aikins Nova Science Publishers, Inc., New York.

[31] Poureslami, I., Nimmon, L., Doyle-Waters, M., Rootman, I., Schulzer, M., Kuramoto, L. and FitzGerald, J.M. (2012) Effectiveness of Video-Based versus Written Educational Interventions on Asthma Self-Management and Inhaler Technique in Punjabi and Chinese Asthma Patients: A Randomized Controlled Trial. Journal of Asthma, 49, 542-551. https://doi.org/10.3109/02770903.2012.682125

[32] Poureslami, I., Kwan, S., Lam, S., Khan, N. and FitzGerald, J.M. (2016) Assessing the Effect of Culturally Specific Audiovisual Educational Interventions on Attaining Self-Management Skills for Chronic Obstructive Pulmonary Disease in Mandarin and Cantonese-Speaking Patients: A Randomized Controlled Trial. International Journal of Chronic Obstructive Pulmonary Disease, 11, 1811-1822.

https://doi.org/10.2147/COPD.S105408

[33] Partridge, M.R. (2000) In What Way May Race, Ethnicity or Culture Influence Asthma Outcomes? Thorax, 55, 175-176. https://doi.org/10.1136/thorax.55.3.175

[34] Bichel, A., Erfle, S., Wiebe, V., et al. (2009) Improving Patient Access to Medical Services: Preventing the Patient from Being Lost in Translation. Healthcare Quarterly, 13, 61-68.

[35] Bertakis, K.D. (1977) The Communication of Information from Physician to Pa- 
tient: A Method for Increasing Patient Retention and Satisfaction. The Journal of Family Practice, 5, 217-222.

[36] Levinson, W., Roter, D.L., Mullooly, J.P., et al. (1997) Physician-Patient Communication. The Relationship with Malpractice Claims among Primary Care Physicians and Surgeons. JAMA, 277, 553-559. https://doi.org/10.1001/jama.1997.03540310051034

[37] Fiese, B.H. and Wamboldt, F.S. (2000) Family Routines, Rituals, and Asthma Management: A Proposal for Family-Based Strategies to Increase Treatment Adherence. Families, Systems and Health, 18, 405-418. https://doi.org/10.1037/h0091864

[38] Hartmann, M., Bäzner, E., Wild, B., Eisler, I. and Herzog, W. (2010) Effects of Interventions Involving the Family in the Treatment of Adult Patients with Chronic Physical Diseases: A Meta-Analysis. Psychotherapy and Psychosomatics, 79, 136148. https://doi.org/10.1159/000286958

[39] Flores, G. (2005) The Impact of Medical Interpreter Services on the Quality of Health Care: A Systematic Review. Medical Care Research and Review, 62, 255-299. https://doi.org/10.1177/1077558705275416

[40] The Lung Association of Ontario: The Ontario Lung Health Action Plan. November 25, 2014, Toronto, Ontario, Canada. https://www.on.lung.ca/document.doc?id=2418

[41] Reece, S., Silka, L., Chao, K. and Phan. P. (2002) Cultural Interpretations of Asthma: Exploring Explanatory Models of Families, Key Informants, and Health Care Providers within the Cambodian Community. 1-18.

[42] Sadatsafavi, M., FitzGerald, J.M., Marra, C. and Lynd, L.D. (2013) Costs and Health Outcomes Associated With Primary vs. Secondary Care after an Asthma-Related Hospitalization: A Population-Based Study. Chest, 144, 428-435. https://doi.org/10.1378/chest.12-2773

[43] Luga, A.O. and McGuire, M.J. (2014) Adherence and Health Care Costs. Risk Management and Healthcare Policy, 7, 35-44.

[44] Enarson, D.A. and Ait-Khaled, N. (1999) Cultural Barriers to Asthma Management [Commentary]. Pediatric Pulmonology, 28, 297-300. https://doi.org/10.1002/(SICI)1099-0496(199910)28:4<297::AID-PPUL9>3.0.CO;2-S

[45] Andersen, M. (1993) Studying across Difference, Race, Class and Gender in Qualitative Research. In: Stanfield, J. II, Ed., Race and Ethnicity in Research Methods, Sage, London, 39-52.

[46] Bhawuk, D. and Triandis, H. (1996) The Role of Culture Theory in the Study of Culture and Intercultural Training. In: Landis, D. and Brislin, R.W., Eds., Handbook of Intercultural Training, Sage, Thousand Oaks, 17-34.

[47] Sudre, P., Jacquemet, S., Uldry, C. and Perneger, T.V. (1999) Objectives, Methods and Content of Patient Education Programmes for Adults with Asthma: Systematic Review of Studies Published between 1979 and 1998. Thorax, 54, 681-687. https://doi.org/10.1136/thx.54.8.681

[48] Torsch, V.L. and Xueqin, M.G. (2000) Cross-Cultural Comparison of Health Perceptions, Concerns, and Coping Strategies among Asian and Pacific Islander American Elders. Qualitative Health Research, 10, 471-489. https://doi.org/10.1177/104973200129118589

[49] Aneas, M.A. and Sandín, M.P. (2009) Intercultural and Cross-Cultural Communication Research: Some Reflections about Culture and Qualitative Methods. Qualitative Social Research, 10, 51.

[50] FitzGerald, J.M. and Poureslami, I. (2014) The Need for Humanomics in the Era of 
Genomics and the Challenge of Chronic Disease Management. Chest, 146, 10-12. https://doi.org/10.1378/chest.13-2817

Submit or recommend next manuscript to SCIRP and we will provide best service for you:

Accepting pre-submission inquiries through Email, Facebook, LinkedIn, Twitter, etc. A wide selection of journals (inclusive of 9 subjects, more than 200 journals)

Providing 24-hour high-quality service

User-friendly online submission system

Fair and swift peer-review system

Efficient typesetting and proofreading procedure

Display of the result of downloads and visits, as well as the number of cited articles

Maximum dissemination of your research work

Submit your manuscript at: http://papersubmission.scirp.org/

Or contact health@scirp.org 